\title{
Effect of elastin-derived peptides on the production of tissue inhibitor of metalloproteinase-1, -2 , and -3 and the ratios in various endothelial cell lines
}

\author{
KRZYSZTOF SIEMIANOWICZ ${ }^{1}$, WIRGINIA LIKUS ${ }^{2}$, TOMASZ FRANCUZ ${ }^{1}$ and WOJCIECH GARCZORZ ${ }^{1}$ \\ Departments of ${ }^{1}$ Biochemistry and ${ }^{2}$ Human Anatomy, School of Medicine, \\ Medical University of Silesia, Katowice 40-752, Poland
}

Received June 8, 2014; Accepted February 5, 2015

DOI: $10.3892 /$ etm.2015.2429

\begin{abstract}
Tissue inhibitors of metalloproteinases (TIMPs) control the activity of metalloproteinases. Elastin-derived peptides (EDPs) are generated as a result of the degradation of elastin fibers. The EDPs bind to the elastin receptor and exert numerous biological effects. The aim of the present study was to compare the production of TIMP-1, TIMP-2 and TIMP-3 and their ratios in human endothelial cells (ECs) derived from three clinically important vascular localizations (coronary arteries, aorta and iliac artery), and evaluate the influence of a well-known EDP, $\kappa$-elastin. The highest concentration of TIMP-1 was identified in the aortic ECs, while the lowest concentration was observed in the ECs derived from the coronary artery. The opposite pattern was observed for TIMP-2 production. When the TIMP-3 concentration was analyzed in the three EC lines, no statistically significant differences were observed. Application of $\kappa$-elastin was found to decrease the TIMP-1 concentration in the aortic ECs, while an increase in the TIMP-1 concentration was observed in the ECs derived from the iliac artery. The most significant decrease in TIMP-2 concentration following $\kappa$-elastin administration was observed in the ECs obtained from the coronary arteries. Furthermore, the highest concentration of $\kappa$-elastin resulted in an increase in TIMP-3 production in the ECs derived from the coronary arteries. The following ratios of the TIMP concentrations were calculated: TIMP-1/TIMP-2, TIMP-1/TIMP-3 and TIMP-2/TIMP-3. Each ratio presented different values for the ECs obtained from the various localizations. In the majority of cases, the addition of $\kappa$-elastin did not significantly change these proportions. Therefore, these indicators may be characteristic features that can be
\end{abstract}

Correspondence to: Dr Krzysztof Siemianowicz, Department of Biochemistry, School of Medicine, Medical University of Silesia, 18 Medyków Street, Katowice 40-752, Poland

E-mail:ksiem@mp.pl

Key words: endothelium, tissue inhibitor of metalloproteinase, elastin-derived peptides used to describe ECs in various clinically important vascular localizations.

\section{Introduction}

Matrix metalloproteinases (MMPs) belong to a family of zinc-dependent endopeptidases that degrade proteins of the extracellular matrix (ECM). MMPs regulate important biological functions, including ECM integrity. The enzymes are expressed in normal and diseased states and participate in vascular and cardiac remodeling, wound healing, tissue resorption and tumor metastasis $(1,2)$. The activity of MMPs is tightly controlled by several inhibitors, including the nonspecific $\alpha_{2}$-macroglobulin and the locally produced specific tissue inhibitors of metalloproteinases (TIMPs). Four human TIMPs have been identified to date, which include TIMP-1, TIMP-2, TIMP-3 and TIMP-4. These proteins constitute a group of small molecules with a mass of 21-28 kDa, which reversibly inhibit the activity of MMPs, binding to them in a $1: 1$ stoichiometric ratio (3). TIMPs function in a tissue environment to neutralize metalloproteinases that have already functioned, thereby preventing excessive and uncontrolled degradation away from the site of MMP production. The role of TIMPs is essential for the homeostasis of the ECM. Although TIMPs share notable homology and structural identity at the protein level, the different forms may vary in their functions. TIMP-1 inhibits angiogenesis by restraining the MMP-9-mediated release of vascular endothelial growth factor (VEGF) from the matrix (4). TIMP-2 has also been shown to present antiangiogenic properties by functioning through MMP inhibition or through direct endothelial cell (EC) binding. In addition, TIMP-2 regulates MMP-14-induced MMP-2 activation by forming a tertiary complex with pro-MMP-2 and its activator, MMP-14, on the cell surface. TIMP-2 can either initiate or restrain the cleavage and subsequent activation of MMP-2 (5). Furthermore, TIMP-2 can bind to cells via interaction with integrin $\alpha 3 \beta 1$ on the surface of human microvascular ECs. This interaction mediates the suppression of VEGF or fibroblast growth factor-mediated cell proliferation (6). TIMP-3 has been shown to promote apoptosis (7).

The endothelium is composed of a single layer of cells that constitute the inner surface of the blood vessels. The 
endothelium functions not only as a barrier between the blood and smooth muscle cells of the blood vessels, but synthesizes and releases various substances with potent multidirectional biological functions. Therefore, the endothelium plays a crucial role in the regulation of vasomotorics and hemostasis. ECs can be a source of the substances involved in angiogenesis and the inflammatory process.

Atherosclerosis is a systemic multiform condition that leads to various diseases depending on the vascular site where the condition is most pronounced. For example, coronary heart disease (CHD), stroke or intermittent claudication may be caused by ischemia in the lower limbs. Furthermore, the aortic wall may be a site of a different dangerous pathology, namely an aortic aneurysm. The American National Cholesterol Education Program, Adult Treatment Panel III, indicates that an abdominal aortic aneurysm should be considered as an equivalent of CHD, which is one of the manifestations of atherosclerosis (8). Endothelium dysfunction, independent of the underlying causes, is the first step leading to the development of atherosclerosis, CHD and other cardiovascular diseases (CVDs) (9).

Elastin-derived peptides (EDPs) are generated as a result of the degradation of elastin fibers. Elastin is a long-lived macromolecule with a very slow turn-over. However, the metabolism of elastin is accelerated in various disease states, such as atherosclerosis, lung emphysema, neoplasm and arthritis (10). Cell responses to EDPs are attributed to the binding of a VGVAPG hexapeptide sequence, which can be detected in insoluble elastin or EDPs. This sequence binds to the elastin receptor and exerts numerous biological effects (11). An experimental study undertaken in our Department has revealed that rabbits receiving EDP injections develop atherosclerosis (12). In addition, disturbances in elastin metabolism, leading to increased serum levels of EDPs, are considered as one of the risk factors of atherosclerosis. Since $\kappa$-elastin is an acknowledged EDP, numerous experiments have evaluated the effect of $\kappa$-elastin on the aorta and EC lines (13-15). In a previous study, the influence of $\kappa$-elastin on the production of MMP-1 and MMP-2 in cultured human EC lines derived from coronary arteries, the iliac artery and aorta was evaluated (16). These localizations in the vascular system are clinically important and present various conditions of blood flow.

The aim of the present study was to compare the production of three TIMPs, TIMP-1, TIMP-2 and TIMP-3, and their concentration ratios in cultured human endothelium derived from three clinically important vascular localizations, namely the coronary arteries, aorta and iliac artery. In addition, the present study evaluated the effect of $\kappa$-elastin on the production of the three TIMPs and their proportions in the three studied endothelial cell lines.

\section{Materials and methods}

Cell culture. EC lines isolated from the human coronary artery, iliac artery and aorta were purchased from Lonza (Basel, Switzerland). The three EC lines were cultured according to the manufacturer's recommendations. Briefly, the cells were maintained in EBM-2 medium with 5\% fetal bovine serum and endothelial cell-specific supplements (insulin-like growth factor, VEGF and heparin; all purchased from Lonza) in a
$95 \% \mathrm{CO}_{2}$ atmosphere at $37^{\circ} \mathrm{C}$. ECs were used in the experiments after 3-4 passages. Following trypsinization, the cells were grown to confluence in 24 -well plates. Subsequently, after serum starvation, the cells were incubated with $\kappa$-elastin (Elastin Products Company, Owensville, MO, USA) at concentrations of $0.1,0.4,1.0,2.5$ or $5.0 \mu \mathrm{g} / \mathrm{ml}$ for $24 \mathrm{~h}$. The cell culture medium was removed, centrifuged for $15 \mathrm{~min}$ at $1,500 \mathrm{x} \mathrm{g}$ and stored at $-70^{\circ} \mathrm{C}$ for subsequent analyses.

ELISA. TIMP-1, TIMP-2 and TIMP-3 concentrations were determined using commercially available kits (R\&D Systems, Inc., Minneapolis, MN, USA) with antibodies against human TIMPs (R\&D Systems, Inc.). The experiment was performed four times with two repeats at each time, producing eight sets of samples for the determination of the evaluated parameters. Each measurement was performed in duplicate and a mean value of the two determinations was calculated.

Statistical analysis. Results are expressed as the mean \pm standard deviation. Prior to the parametric analyses, normal distribution was verified with the Shapiro-Wilk test. In addition, the homogeneity of variance was analyzed using Bartlett's test. The statistical significance of the differences between groups was calculated using a parametric one-way analysis of variance test for data with a normal distribution, assuming homogeneity and heterogeneity of variances, followed by the Tukey-Kramer test. The Kruskal-Wallis rank test was applied in the case of non-normality of distribution, which was followed by the Steel-Dwass test. Dunnett's test or Steel's test was used to compare each group with the control. $\mathrm{P} \leq 0.05$ was considered to indicate a statistically significant difference. The statistical software Statistica 10.0 (Statsoft, Tulsa, Oklahoma, USA) was used in this analysis.

\section{Results}

Concentration of TIMPs in the various EC lines. The highest concentration of TIMP-1 was observed in the aortic endothelium, while the lowest concentration was detected in the endothelium derived from the coronary artery $(\mathrm{P}<0.01)$. In the endothelium derived from the iliac artery, a statistically significantly decrease was observed in the TIMP-1 concentration when compared with the aortic ECs $(\mathrm{P}<0.01)$, while a statistically significant increase was observed when compared with the coronary artery ECs $(\mathrm{P}<0.01$; Fig. 1A). The opposite pattern of dependence was observed in the case of TIMP-2. TIMP-2 reached the highest concentration in the cell culture medium from the coronary artery endothelium, while the lowest concentration was observed in the aortic ECs $(\mathrm{P}<0.001)$. In addition, in the ECs derived from the iliac artery, a statistically significant decrease was observed in the concentration of TIMP-2 when compared with the ECs derived from the coronary artery $(\mathrm{P}<0.01)$, while a statistically significant increase was observed when compared with the aortic ECs $(\mathrm{P}<0.001$; Fig. 1B). However, when the TIMP-3 concentration was analyzed in the three EC lines, no statistically significant differences were identified (Fig. 1C).

Effect of $\kappa$-elastin on TIMP-1 production. Following the addition of $\kappa$-elastin at concentrations between 0.4 and $5.0 \mu \mathrm{g} / \mathrm{ml}$ to the cell culture of aortic ECs, a statistically significant decrease 
A

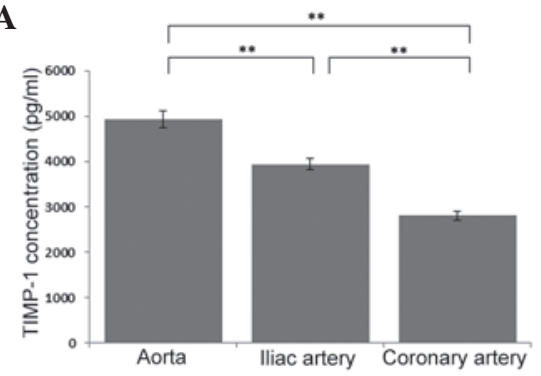

B

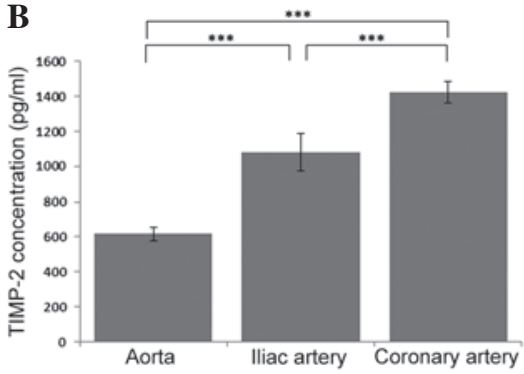

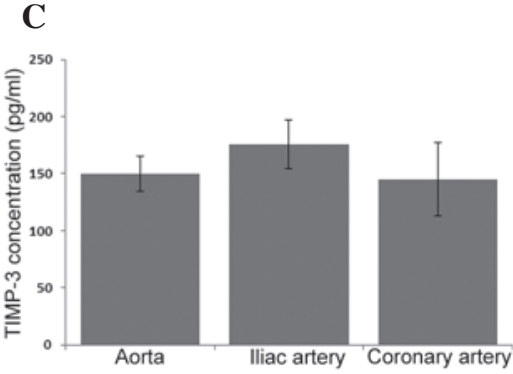

Figure 1. Concentration of (A) TIMP-1, (B) TIMP-2 and (C) TIMP-3 in the various endothelial cell lines (controls). Values are presented as the mean \pm standard deviation. ${ }^{* *} \mathrm{P}<0.01$ and ${ }^{* * * *} \mathrm{P}<0.001$, compared between groups. TIMP, tissue inhibitor of metalloproteinase.

A
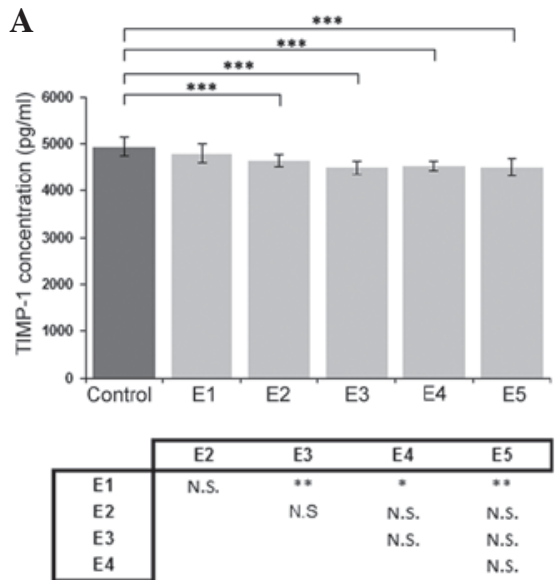

B
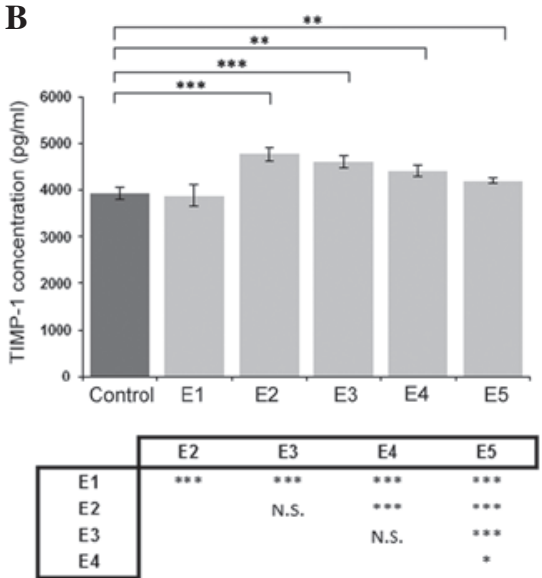

C
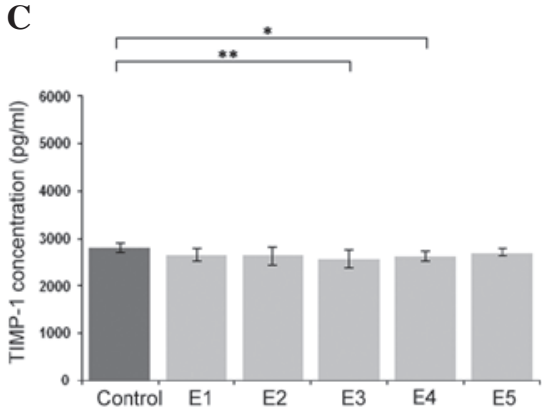

\begin{tabular}{|l|cccc|}
\cline { 2 - 5 } \multicolumn{1}{c|}{} & E2 & E3 & E4 & E5 \\
\hline E1 & N.S. & N.S & N.S & N.S \\
E2 & & N.S & N.S. & N.S. \\
E3 & & & N.S. & N.S. \\
E4 & & & & N.S. \\
\hline
\end{tabular}

Figure 2. Effects of various concentrations of $\kappa$-elastin on the TIMP-1 concentration in human endothelial cells derived from the (A) aorta, (B) iliac artery and (C) coronary artery. E1, E2, E3, E4 and E5, $\kappa$-elastin concentrations of $0.1,0.4,1.0,2.5$ and $5.0 \mu \mathrm{g} / \mathrm{ml}$, respectively. Values are presented as the mean \pm standard deviation. ${ }^{*} \mathrm{P}<0.05,{ }^{* *} \mathrm{P}<0.01$ and ${ }^{* * *} \mathrm{P}<0.001$, compared between groups. N.S., not significant; TIMP, tissue inhibitor of metalloproteinase.
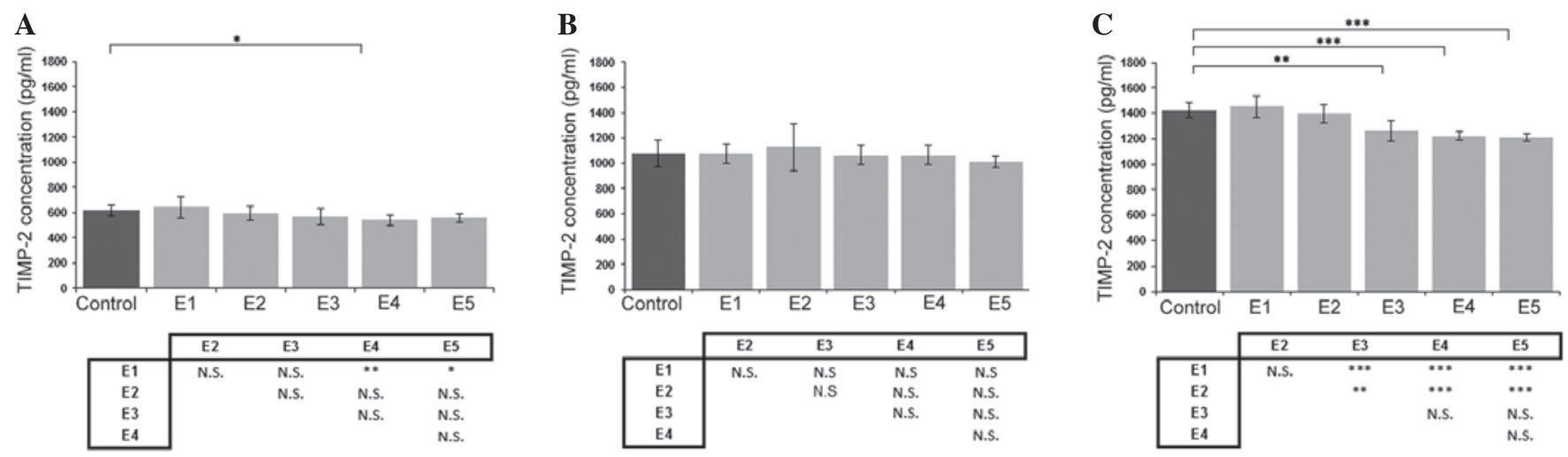

Figure 3. Effects of various concentrations of $\kappa$-elastin on the TIMP-2 concentration in human endothelial cells derived from the (A) aorta, (B) iliac artery and (C) coronary artery. E1, E2, E3, E4 and E5, $\mathrm{k}$-elastin concentrations of $0.1,0.4,1.0,2.5$ and $5.0 \mu \mathrm{g} / \mathrm{ml}$, respectively. Values are presented as the mean \pm standard deviation. ${ }^{*} \mathrm{P}<0.05,{ }^{* *} \mathrm{P}<0.01$ and ${ }^{* * * *} \mathrm{P}<0.001$, compared between groups. N.S., not significant; TIMP, tissue inhibitor of metalloproteinase.

in the TIMP-1 concentration was observed $(\mathrm{P}<0.001$; Fig. $2 \mathrm{~A})$. However, in the cell culture of the coronary artery-derived ECs, a higher concentration of $\kappa$-elastin $(1.0$ and $2.5 \mu \mathrm{g} / \mathrm{ml})$ was required to cause a statistically significant reduction in the TIMP-1 concentration (Fig. 2C). The opposite effect of $\kappa$-elastin on TIMP-1 production was observed in the ECs derived from the iliac artery, where an increase in the TIMP-1 concentration was detected at lower $\kappa$-elastin concentrations. The increase was most pronounced at a $\kappa$-elastin concentration of $0.4 \mu \mathrm{g} / \mathrm{ml}$, which resulted in $\sim 23 \%$ increase in the TIMP-1 concentration in the cell culture medium (Fig. 2B).

Effect of $\kappa$-elastin on TIMP-2 production. Evaluation of the effect of $\kappa$-elastin on TIMP-2 concentration revealed that a decrease in concentration was most pronounced in the ECs obtained from the coronary arteries (Fig. 3C). With regard to the aortic ECs, only a concentration of $1.0 \mu \mathrm{g} / \mathrm{ml}$ caused a statistically significant reduction in TIMP-2 production 
Table I. Concentration ratios of TIMPs in the various EC lines.

\begin{tabular}{|c|c|c|c|}
\hline EC line & TIMP-1/TIMP-2 & TIMP-2/TIMP-3 & TIMP-1/TIMP-3 \\
\hline \multicolumn{4}{|l|}{ Control } \\
\hline Aorta & 7.0 & 4.0 & 33.0 \\
\hline Iliac artery & 3.6 & 6.1 & 22.0 \\
\hline Coronary artery & 1.9 & 9.8 & 19.5 \\
\hline \multicolumn{4}{|l|}{ E1 } \\
\hline Aorta & 1.9 & 3.7 & 28.2 \\
\hline Iliac artery & 3.5 & 6.4 & 22.9 \\
\hline Coronary artery & 1.8 & 9.3 & 17.0 \\
\hline \multicolumn{4}{|l|}{ E2 } \\
\hline Aorta & 8.6 & 3.6 & 28.0 \\
\hline Iliac artery & 4.2 & 6.3 & 27.0 \\
\hline Coronary artery & 1.8 & 10.4 & 19.7 \\
\hline \multicolumn{4}{|l|}{ E3 } \\
\hline Aorta & 7.9 & 3.3 & 26.2 \\
\hline Iliac artery & 4.3 & 5.5 & 24.0 \\
\hline Coronary artery & 2.0 & 8.5 & 17.3 \\
\hline \multicolumn{4}{|l|}{$\mathrm{E} 4$} \\
\hline Aorta & 8.3 & 3.5 & 29.8 \\
\hline Iliac artery & 4.1 & 5.9 & 25.0 \\
\hline Coronary artery & 2.0 & 6.9 & 14.7 \\
\hline \multicolumn{4}{|l|}{ E5 } \\
\hline Aorta & 8.0 & 4.0 & 33.0 \\
\hline Iliac artery & 4.0 & 6.4 & 26.6 \\
\hline Coronary artery & 2.2 & 6.0 & 13.2 \\
\hline
\end{tabular}

Control cells were unstimulated. E1, E2, E3, E4 and E5, $\kappa$-elastin concentrations of 0.1, 0.4, 1.0, 2.5 and 5.0 $\mu \mathrm{g} / \mathrm{ml}$, respectively. TIMP, tissue inhibitor of metalloproteinase; EC, endothelial cell.

A

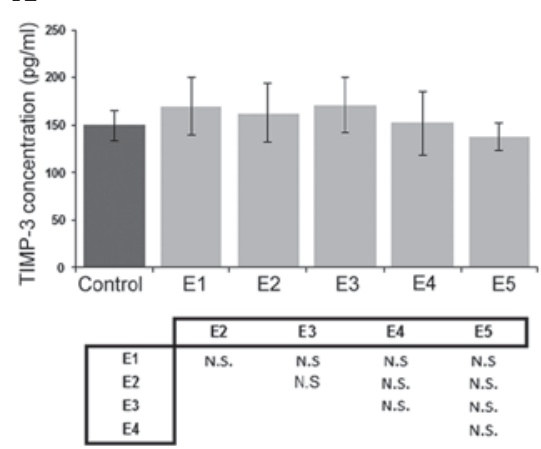

$\mathbf{B}$

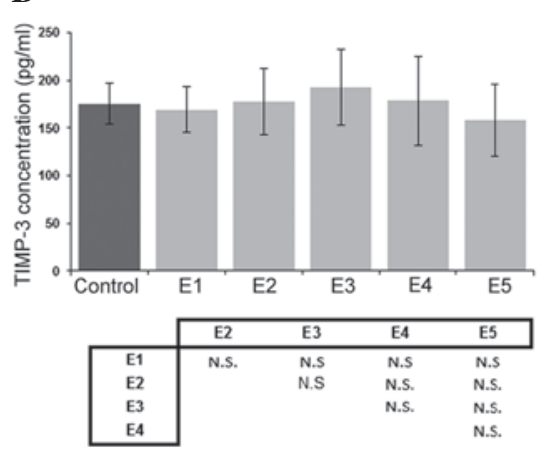

C

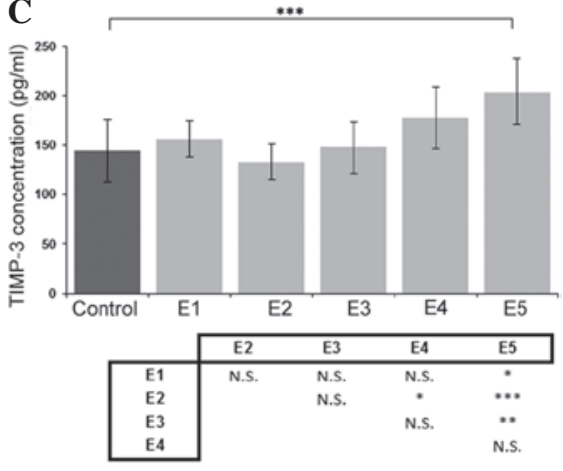

Figure 4. Effects of various concentrations of $\kappa$-elastin on the TIMP-3 concentration in human endothelial cells derived from the (A) aorta, (B) iliac artery and (C) coronary artery. E1, E2, E3, E4 and E5, $\kappa$-elastin concentrations of $0.1,0.4,1.0,2.5$ and $5.0 \mu \mathrm{g} / \mathrm{ml}$, respectively. Values are presented as the mean \pm standard deviation. ${ }^{*} \mathrm{P}<0.05,{ }^{* *} \mathrm{P}<0.01$ and ${ }^{* * * *} \mathrm{P}<0.001$, compared between groups. N.S., not significant; TIMP, tissue inhibitor of metalloproteinase.

(Fig. 3A). In addition, $\kappa$-elastin did not cause any statistically significant changes in the TIMP-2 concentration in the ECs derived from the iliac artery (Fig. 3B).

Effect of $\kappa$-elastin on TIMP-3 production. When the effect of $\kappa$-elastin on the concentration of TIMP-3 was analyzed, no statistically significant differences were detected in the cell lines derived from the aorta or iliac artery (Fig. 4A and B). However, the highest studied concentration of $\kappa$-elastin caused a statistically significant increase in TIMP-3 production in the ECs derived from the coronary arteries (Fig. 4C).

Effect of $\kappa$-elastin on the concentration of TIMPs in the various EC lines. Comparisons of the effect of different concentrations 
of $\kappa$-elastin revealed no statistically significant differences in the TIMP-1 concentration in the ECs derived from the coronary arteries, in the TIMP- 2 concentration in the ECs derived from the iliac artery and in the TIMP-3 concentration in the aortic ECs and ECs derived from the iliac artery (Figs. 2C, $3 \mathrm{~B}, 4 \mathrm{~A}$ and $\mathrm{B})$.

The most pronounced effect of $\kappa$-elastin on the production of TIMP-1 was observed in the ECs derived from the aorta and iliac artery. However, with regard to TIMP-2, the most pronounced effect was observed in the ECs derived from the coronary artery.

TIMP concentration ratios. The following ratios of TIMP concentrations were calculated: TIMP-1/TIMP-2, TIMP-1/TIMP-3 and TIMP-2/TIMP-3 (Table I). Each ratio presented a different value in the ECs obtained from the various localizations. For example, the TIMP-1/TIMP-2 ratio was equal to seven in the unstimulated aortic ECs, while the ratio was determined to be $\sim 3.6$ in the iliac artery ECs and $\sim 1.9$ in the coronary artery ECs. In the majority of cases, the addition of $\kappa$-elastin did not significantly change these proportions. The preservation of the magnitude of these TIMP ratios was observed for all three TIMP ratios and for all three studied EC lines. In addition, the stimulatory and inhibitory effects of $\kappa$-elastin were not found to significantly alter or reverse these proportions.

\section{Discussion}

EDPs have been demonstrated to be risk factors for atherosclerosis. Robinet et al (17) observed that EDPs containing the VGVAPG motif accelerated angiogenesis by inducing the angiogenic phenotype of ECs.

TIMP-1 has attracted increasing attention as a possible novel marker of endothelium dysfunction. A number of studies have focused on evaluating the level of TIMP-1 in blood samples obtained from patients with various risk factors of atherosclerosis and CVD. Mieczkowska et al (18) observed that females with metabolic syndrome had statistically higher blood levels of TIMP-1. In addition, TIMP-1 was found to positively correlate with a variety of risk factors, such as body mass index, waist to hip ratio, waist circumference, fasting glucose level and triglyceride concentration, while negatively correlating with the high-density lipoprotein cholesterol level. Furthermore, de la Sierra and Larrousse (19) found that TIMP-1 may be a marker of endothelial dysfunction in high-risk hypertensive patients. TIMP-1 was shown to have a significant inverse correlation with maximal endothelium-dependent vasodilatation. Ragino et al (20) compared the blood levels of TIMP-1 in patients with unstable and stable atherosclerotic plaques in the coronary arteries. Circulating TIMP-1 levels were revealed to be lower in the individuals with unstable plaques. The aforementioned studies do not present convergent results, which may be due to the methodology of these studies. Serum levels of TIMP-1 are a sum of the production by ECs in the whole vascular system. The results of the present study indicated that the same stimulator, $\kappa$-elastin, may have a divergent influence on the endothelium depending on the localization in the circulatory system. For example, a stimulatory effect on TIMP-1 production was observed in the ECs derived from the iliac artery, while an inhibitory effect was observed in the EC lines obtained from the aorta and coronary arteries. In the case of TIMP-2 production, in the ECs obtained from the aorta and iliac artery, the effect of $\kappa$-elastin appeared to be neutral.

In an experimental model, the induction of a murine femoral artery injury caused an initial decrease in TIMP-1 and TIMP-2 expression and activity levels during the first five days, with subsequent restoration of the expression and activity levels (21). However, in a similar preceding experiment, no changes in TIMP-1 and TIMP-2 expression and activity levels were observed (22). Moore et al (23) observed that in rats subjected to oxidative stress, plasma levels of TIMP-1 may serve as an early marker of endothelium dysfunction.

Using an experimental model, Misra et al observed that murine fibroblasts cultured in hypoxemic conditions exhibited increased expression levels of TIMP-1 and TIMP-2; however, these changes were not simultaneous. An augmentation in TIMP-1 expression preceded the increased expression of TIMP-2 (24). Furthermore, Mammi et al observed that in patients with heart failure, serum levels of TIMP-1 can be decreased by physical exercise (25). Notably, Ramirez Correa et al (26) used local TIMP-1 gene transfer and achieved a significant reduction of restenosis in human coronary smooth muscle cells.

EDPs have been shown to promote angiogenesis (17), a process that is involved in a variety of pathological and physiological conditions. Angiogenesis plays a pivotal role in postischemic neovascularization of the myocardium. The results of the present study indicated that EDPs may increase the production of TIMPs. The results of these two studies $(24,25)$ and the present study can make one raise a question about an influence of EDPs on coronary arteries, whether EDPs may be deleterious or beneficial in certain circumstances. TIMP-2 also exhibits angioinhibitory properties, which comprise several mechanisms, including MMP inhibition or direct EC binding (27). In addition, the results of the present study indicated that TIMP-1 and TIMP-2 presented various production profiles in unstimulated ECs, and their production differed depending on the vasculature localization. However, the effect of $\kappa$-elastin preserved the production proportion in each cell line from the various localizations in the vascular bed. Therefore, the TIMP concentrations proportion may be a characteristic feature of ECs in various arteries. The calculated TIMP ratios support the observation that the endothelium does not constitute a uniform pool of cells with the same biological characteristics. The biochemical properties can vary depending on their localization in the cardiovascular system and the different characteristics of blood flow in these places. Therefore, the observations of the present study have an important implication. While studying EC biology, biochemistry and their response to various stimuli, the localization of the obtained ECs should be taken into consideration.

TIMP-3 is considered to be a major regulator of angiogenesis. The protein can block the binding of VEGF to its receptor, VEGFR-2, and this function is independent of the MMP inhibitory effect (28). In addition, TIMP-3 can induce vascular cell apoptosis. Vacek et al (29) used a mouse experimental model and found that a mechanical injury of the carotid artery led to an increase in the TIMP-3 level in the injured artery. Similar results were obtained by Basu et al (30). In mice 
with chronic hyperhomocysteinemia, an increase in TIMP-3 gene expression was observed in the aorta. Furthermore, in an additional study, when a cigarette smoke extract was used as an evaluated risk factor, a decreased secretion of TIMP-3 was detected in the rabbit endothelium (31). TIMP-3 has been analyzed as a factor for gene therapy, and overexpression of TIMP-3 has been shown to result in a sustained retardation of vein graft intimal thickening (32). In the present study, TIMP-3 production appeared to be the most resistant to the influence of $\kappa$-elastin as a modulator.

In conclusion, the present study demonstrated that ECs from various parts of the vascular system present a different production with regard to the three analyzed TIMPs. These cells also differ in their response to $\kappa$-elastin, applied as a modulator. Therefore, the observations of the current study indicate that the endothelium can adapt to various conditions of blood flow in different sections of the vascular system and modulate the production of important molecules involved in ECM homeostasis.

A number of studies have calculated and analyzed various TIMP/MMP ratios $(21,25)$. However, the present study was the first to calculate the ratios for three TIMP indicators: TIMP-1/TIMP-2, TIMP-1/TIMP-3 and TIMP-2/TIMP-3. The results demonstrated that ECs in various localizations had their own specific values of these ratios that are preserved when a risk factor, such as $\kappa$-elastin, influences the endothelium. Therefore, these ratio indicators may be characteristic features used to describe ECs in various clinically important vascular localizations. Subsequently, the biological differences of ECs derived from various localizations in the cardiovascular system should be taken into consideration in further studies evaluating the function of ECs and their response to various stimuli.

\section{References}

1. Ganea E, Trifan M, Laslo AC, Putina G and Cristescu C: Matrix metalloproteinases: useful and deleterious. Biochem Soc Trans 35: 689-691, 2007.

2. Raffetto JD and Khalil RA: Matrix metalloproteinases and their inhibitors in vascular remodeling and vascular disease. Biochem Pharmacol 75: 346-359, 2008.

3. Troeberg L and Nagase H: Analysis of TIMP expression and activity. Methods Mol Med 135: 251-267, 2007.

4. Stetler-Stevenson WG: The tumor microenvironment: regulation by MMP-independent effects of tissue inhibitor of metalloproteinases-2. Cancer Metastasis Rev 27: 57-66, 2008.

5. Shen Q, Lee ES, Pitts RL, Wu MH and Yuan SY: Tissue inhibitor of metalloproteinase-2 regulates matrix metalloproteinase-2-mediated endothelial barrier dysfunction and breast cancer cell transmigration through lung microvascular endothelial cells. Mol Cancer Res 8: 939-951, 2010.

6. Seo DW, Li H, Guedez L, Wingfield PT, et al: TIMP-2 mediated inhibition of angiogenesis: an MMP-independent mechanism Cell 114: 171-180, 2003.

7. Kallio JP, Hopkins-Donaldson S, Baker AH and Kähäri VM: TIMP-3 promotes apoptosis in nonadherent small cell lung carcinoma cells lacking functional death receptor pathway. Int J Cancer 128: 991-996, 2011.

8. Grundy SM; National Cholesterol Education Program (NCEP) - The National Cholesterol Guidelines in 2001, Adult Treatment Panel (ATP) III: Approach to lipoprotein management in 2001 National Cholesterol Guidelines. Am J Cardiol 90: $11 \mathrm{i}-21 \mathrm{i}, 2002$

9. Ross R: Atherosclerosis - an inflammatory disease. N Engl J Med 340: 115-126, 1999.

10. Hornebeck W and Robert L: Elastase-like enzymes in aortas and human breast carcinomas: quantitative variations with age and pathology. Adv Exp Med Biol 79: 145-164, 1977.
11. Gayral S, Garnotel R, Castaing-Berthou A, et al: Elastin-derived peptides potentiate atherosclerosis through the immune Neu1-PI3K $\gamma$ pathway. Cardiovasc Res 102: 118-127, 2014.

12. Gmiński J and Drózdz M: Succinyl trialanine p-nitroanilide hydrolytic activities in plasma and the aorta of rabbits experimentally immunized with soluble elastin. Exp Pathol 43: 37-40, 1991.

13. Faury G, Ristori MT, Verdetti J, Jacob MP and Robert L: Effect of elastin peptides on vascular tone. J Vasc Res 32: 112-119, 1995.

14. Faury G, Garnier S, Weiss AS, et al: Action of tropoelastin and synthetic elastin sequences on vascular tone and on free $\mathrm{Ca}^{2+}$ level in human vascular endothelial cells. Circ Res 82: 328-336, 1998.

15. Robert L, Labat-Robert J and Robert AM: Genetic, epigenetic and posttranslational mechanisms of aging. Biogerontology 11: 387-399, 2010.

16. Siemianowicz K, Gminski J, Goss M, Francuz T, Likus W, Jurczak T and Garczorz W: Influence of elastin-derived peptides on metalloprotease production in endothelial cells. Exp Ther Med 1: 1057-1060, 2010.

17. Robinet A, Fahem A, Cauchard J-H, et al: Elastin-derived peptides enhance angiogenesis by promoting endothelial cell migration and tubulogenesis through upregulation of MT1-MMP. J Cell Sci 118: 343-356, 2005

18. Mieczkowska J, Mosiewicz J, Barud W and Kwaśniewski W: Changes in the activity of connective tissue matrix enzymes in the metabolic syndrome. Arch Med Sci 7: 634-641, 2011.

19. de la Sierra A and Larrousse M: Endothelial dysfunction is associated with increased levels of biomarkers in essential hypertension. J Hum Hypertens 24: 373-379, 2010.

20. Ragino IuI, Cherniavskiı̌ AM, Polonskaia IaV, Volkov AM, Kashtanova EV, Tsymbal SIu and Polovnikova EM: Inflammatory-destructive biomarkers of atherosclerotic plaques instability. Study of arterial wall and blood. Kardiologiia 52: 37-41, 2012 (In Russian).

21. Zou Y, Qi Y, Roztocil E and Davies MG: Patterns of gelatinase activation induced by injury in the murine femoral artery. J Surg Res 154: 135-142, 2009.

22. Zou Y, Fu Y and Davies MG: Role for G $\beta \gamma$ G-proteins in protease regulation during remodeling of the murine femoral artery. J Surg Res 178: 40-47, 2013.

23. Moore R, Hawley A, Sigler R, Farris D, Wrobleski S, Ramacciotti E and Myers D: Tissue inhibitor of metalloproteinase-1 is an early marker of acute endothelial dysfunction in a rodent model of venous oxidative injury. Ann Vasc Surg 23: 498-505, 2009.

24. Misra S, Fu AA, Misra KD, Shergill UM, Leof EB and Mukhopadhyay D: Hypoxia-induced phenotypic switch of fibroblasts to myofibroblasts through a matrix metalloproteinase 2/tissue inhibitor of metalloproteinase-mediated pathway: implications for venous neointimal hyperplasia in hemodialysis access. J Vasc Interv Radiol 21: 896-902, 2010.

25. Mammi C, la Sala A, Volterrani M, et al: Exercise training reduces serum capacity to induce endothelial cell death in patients with chronic heart failure. Eur J Heart Fail 13: 642-650, 2011.

26. Ramirez Correa GA, Zacchigna S, Arsic N, Zentilin L, Salvi A, Sinagra $\mathrm{G}$ and Giacca M: Potent inhibition of arterial intimal hyperplasia by TIMP1 gene transfer using AAV vectors. Mol Ther 9: 876-884, 2004.

27. Seo DW, Saxinger WC, Guedez L, Cantelmo AR, Albini A and Stetler-Stevenson WG: An integrin-binding N-terminal peptide region of TIMP-2 retains potent angio-inhibitory and anti-tumorigenic activity in vivo. Peptides. 32: 1840-1848, 2011.

28. Qi JH, Ebrahem Q, Ali M, et al: Tissue inhibitor of metalloproteinases-3 peptides inhibit angiogenesis and choroidal neovascularization in mice. PLoS One 8: e55667, 2013.

29. Vacek TP, Gillespie W, Tyagi N, Vacek JC and Tyagi SC: Hydrogen sulfide protects against vascular remodeling from endothelial damage. Amino Acids 39: 1161-1169, 2010.

30. Basu P, Qipshidze N, Sen U, Givvimani S, Munjal C, Mishra PK and Tyagi SC: Chronic hyperhomocysteinemia causes vascular remodelling by instigating vein phenotype in artery. Arch Physiol Biochem 117: 270-282, 2011.

31. Lemaître V, Dabo AJ and D'Armiento J: Cigarette smoke components induce matrix metalloproteinases-1 in aortic endothelial cells through inhibition of mTOR signaling. Toxicol Sci 123: 542-549, 2011.

32. George SJ, Wan S, Hu J, MacDonald R, Johnson JL and Baker AH: Sustained reduction of vein graft neointima formation by ex vivo TIMP-3 gene therapy. Circulation 124 (Suppl): S135-S142, 2011. 